\title{
Effect of in-plane shear strain on phase states and dielectric properties of epitaxial ferroelectric thin films
}

A. G. Zembilgotov, U. Böttger, and R. Waser

Citation: Journal of Applied Physics 104, 054118 (2008);

View online: https://doi.org/10.1063/1.2976347

View Table of Contents: http://aip.scitation.org/toc/jap/104/5

Published by the American Institute of Physics

\section{Articles you may be interested in}

Temperature-strain phase diagram for $\mathrm{BaTiO}_{3}$ thin films

Applied Physics Letters 88, 072905 (2006); 10.1063/1.2172744

Thermodynamic theory of $\mathrm{PbTiO}_{3}$

Journal of Applied Physics 62, 3331 (1998); 10.1063/1.339293

Ferroelectric, pyroelectric, and piezoelectric properties of a photovoltaic perovskite oxide

Applied Physics Letters 110, 063903 (2017); 10.1063/1.4974735

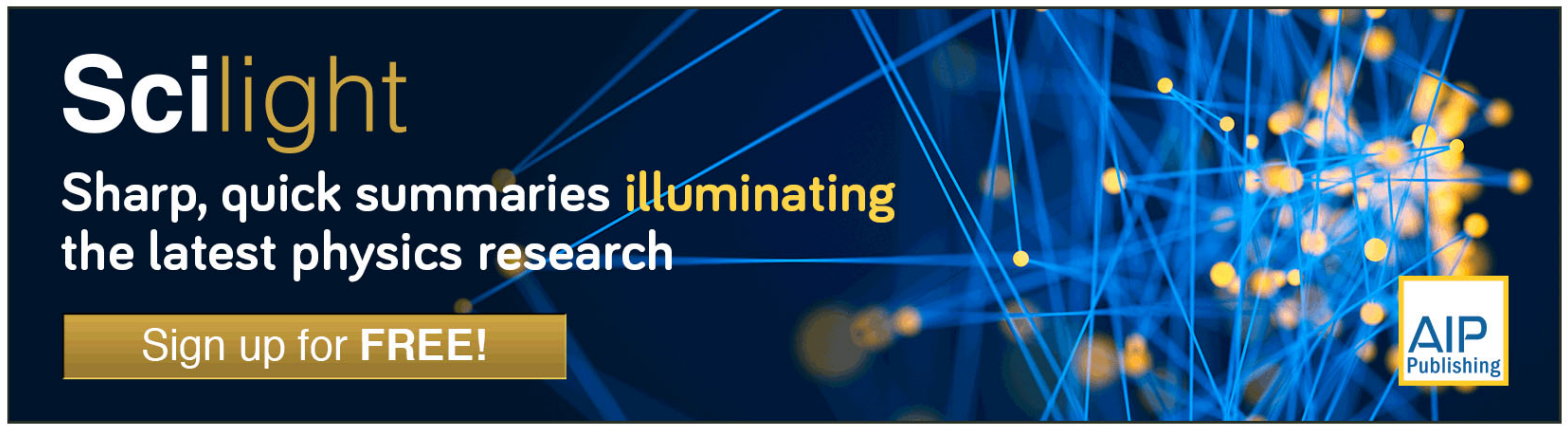




\title{
Effect of in-plane shear strain on phase states and dielectric properties of epitaxial ferroelectric thin films
}

\author{
A. G. Zembilgotov, ${ }^{\text {a) }}$ U. Böttger, and R. Waser ${ }^{\text {b) }}$ \\ Institut für Werkstoffe der Elektrotechnik, RWTH Aachen University of Technology, \\ D-52056 Aachen, Germany
}

(Received 16 September 2007; accepted 8 July 2008; published online 12 September 2008)

\begin{abstract}
A modified thermodynamic potential based on the eight-order Landau-Devonshire polynomial is derived for ferroelectric thin films grown on dissimilar substrates that induce anisotropic tensile/ compressive strains and the shear strain in the film plane. The effect of the shear strain on the ferroelectric phase transition occurring in considered films is analyzed theoretically. It is shown that the application of the shear strain suppresses the formation of ferroelectric $c$ phase and raises the temperature of $a a^{*}$ phase formation. The directional dependence of in-plane dielectric permittivity of a ferroelectric film is also calculated and compared with the dependence observed in $\left(\mathrm{Ba}_{0.60} \mathrm{Sr}_{0.40}\right) \mathrm{TiO}_{3}$ films deposited on $\mathrm{NdGaO}_{3}$. (C) 2008 American Institute of Physics.
\end{abstract}

[DOI: $10.1063 / 1.2976347$ ]

\section{INTRODUCTION}

During the past decade epitaxial ferroelectric thin films are of great interest due to their numerous applications in the fields of microelectronics and micromechanics. The physical properties of single-crystal ferroelectric films are intensively studied experimentally ${ }^{1-8}$ and theoretically. ${ }^{9-25}$ The earlier theoretical studies were restricted to thin films grown on (001)-oriented cubic substrates that induce the strain field isotropic in the film plane. However, other substrates (e.g., orthorhombic) generally will induce the strain field, which is anisotropic in the film plane. The recent experimental studies of epitaxial films grown on orthorhombic substrates have shown that the substrate-induced strain anisotropy may significantly affect the dielectric properties of the ferroelectric layer. ${ }^{7,26,27}$ This fact caused the development of thermodynamic theory of ferroelectric thin films with the anisotropic tensile/compressive strains and the shear deformation acting in the film plane. ${ }^{19}$ Further theoretical studies were devoted to epitaxial films under anisotropic biaxial strains without the shear component. ${ }^{20,22-25}$ Nevertheless, the shear strain acting in the plane of interface might emerge in some special types of epitaxy, for example, if the film and the substrate form "coincidence superlattice." 28 In the present paper we extend the developed thermodynamic theory ${ }^{19}$ and use it to study the effect of the in-plane shear strain on the phase states and dielectric properties of ferroelectric thin films.

In Sec. II, a general expression is derived for the thermodynamic potential $\widetilde{G}$ of a single domain (001)-oriented epitaxial thin film grown on a dissimilar substrate that induces anisotropic biaxial strains and the shear deformation within the film. In contrast to the previous treatment of the problem, ${ }^{19}$ the eight-order elastic Gibbs function was used to construct the modified thermodynamic potential. The influ-

\footnotetext{
${ }^{a)}$ Permanent address: Institute of Trade and Economics, 194021 St. Petersburg, Russia. Electronic mail: az@domain.ioffe.rssi.ru.

b) Also at Institut für Festkörperforschung, Forschungszentrum Jülich, D-52425 Jülich, Germany.
}

ence of misfit strains on the temperature of ferroelectric phase transition is considered in Sec. III. Here we also characterize the different crystallographic phases forming during the ferroelectric phase transformation under various misfit strains. In Sec. IV, the phase maps indicating the stability ranges of various phase states of $\mathrm{BaTiO}_{3}$ (BT) and $\mathrm{Pb}(\mathrm{Zr}, \mathrm{Ti}) \mathrm{O}_{3}$ (PZT) epitaxial films are presented. In Sec. V, we obtain a general expression for in-plane dielectric permittivity of ferroelectric films and describe the characteristic features of its directional dependence. The results of our numerical calculations performed for BT epitaxial films are also reported here. Finally, the main conclusions of this study are formulated in Sec. VI.

\section{THERMODYNAMIC POTENTIAL OF A FERROELECTRIC FILM UNDER ANISOTROPIC IN-PLANE STRAINS AND IN-PLANE SHEAR DEFORMATION}

In this section we develop a modified thermodynamic potential based on an eight-order polynomial to find the equilibrium thermodynamic states of single domain epitaxial thin films grown in the (001)-oriented cubic paraelectric phase on a dissimilar substrate. In general, the substrate induces two different in-plane strains, $u_{1}$ and $u_{2}$, and a shear deformation $u_{6}$ in the film. (We use the Voigt matrix notation and the reference frame with the $x_{3}$ axis orthogonal to the film surfaces.) The strain field inside the film is taken to be homogeneous in our theory. Since the in-plane strains are governed by a thick substrate, they can be regarded as fixed parameters of the film/substrate system: $u_{1}=u_{m 1}, u_{2}=u_{m 2}$, and $u_{6}=u_{m 6}$. The misfit strains $u_{m 1}, u_{m 2}$, and $u_{m 6}$ can be calculated as $u_{m 1}=\left(a_{1}-a_{0}\right) / a_{0}, u_{m 2}=\left(a_{2}-a_{0}\right) / a_{0}$, and $u_{m 6}$ $=\gamma-\pi / 2$, where $a_{0}$ is the equivalent cubic cell constant of the free standing film and $a_{1}, a_{2}$, and $\gamma$ are the in-plane lattice parameters of the epitaxial film.

To correctly take into account the mixed boundary conditions existing in epitaxial film, one should use the modified thermodynamic potential derived from the standard elastic 
Gibbs function $G$ (Refs. 29 and 30) by means of Legendre transformation. ${ }^{19}$ Applying this procedure to the eight-order Landau-Devonshire polynomial ${ }^{30}$ we obtain the following expression for the desired potential $\widetilde{G}$

$$
\begin{aligned}
\widetilde{G}= & \alpha_{1}^{*} P_{1}^{2}+\alpha_{2}^{*} P_{2}^{2}+\alpha_{3}^{*} P_{3}^{2}+\alpha_{6}^{*} P_{1} P_{2}+\alpha_{11}^{*}\left(P_{1}^{4}+P_{2}^{4}\right) \\
& +\alpha_{33}^{*} P_{3}^{4}+\alpha_{13}^{*}\left(P_{1}^{2}+P_{2}^{2}\right) P_{3}^{2}+\alpha_{12}^{*} P_{1}^{2} P_{2}^{2} \\
& +\alpha_{111}\left(P_{1}^{6}+P_{2}^{6}+P_{3}^{6}\right)+\alpha_{112}\left[P_{1}^{4}\left(P_{2}^{2}+P_{3}^{2}\right)+P_{2}^{4}\left(P_{1}^{2}+P_{3}^{2}\right)\right. \\
& \left.+P_{3}^{4}\left(P_{1}^{2}+P_{2}^{2}\right)\right]+\alpha_{123} P_{1}^{2} P_{2}^{2} P_{3}^{2}+\alpha_{111}\left(P_{1}^{8}+P_{2}^{8}+P_{3}^{8}\right) \\
& +\alpha_{1112}\left[P_{1}^{6}\left(P_{2}^{2}+P_{3}^{2}\right)+P_{2}^{6}\left(P_{1}^{2}+P_{3}^{2}\right)+P_{3}^{6}\left(P_{1}^{2}+P_{2}^{2}\right)\right] \\
& +\alpha_{1122}\left(P_{1}^{4} P_{2}^{4}+P_{2}^{4} P_{3}^{4}+P_{1}^{4} P_{3}^{4}\right)+\alpha_{1123}\left(P_{1}^{4} P_{2}^{2} P_{3}^{2}+P_{2}^{4} P_{3}^{2} P_{1}^{2}\right. \\
& \left.+P_{3}^{4} P_{1}^{2} P_{2}^{2}\right)+\frac{\left[s_{11}\left(u_{m 1}^{2}+u_{m 2}^{2}\right)-2 s_{12} u_{m 1} u_{m 2}\right]}{2\left(s_{11}^{2}-s_{12}^{2}\right)}+\frac{u_{m 6}^{2}}{2 s_{44}}
\end{aligned}
$$

where

$$
\alpha_{1}^{*}=\alpha_{1}+\frac{u_{m 1}\left(Q_{12} s_{12}-Q_{11} s_{11}\right)+u_{m 2}\left(Q_{11} s_{12}-Q_{12} s_{11}\right)}{\left(s_{11}^{2}-s_{12}^{2}\right)},
$$

$$
\alpha_{2}^{*}=\alpha_{1}+\frac{u_{m 2}\left(Q_{12} s_{12}-Q_{11} s_{11}\right)+u_{m 1}\left(Q_{11} s_{12}-Q_{12} s_{11}\right)}{\left(s_{11}^{2}-s_{12}^{2}\right)}
$$

$$
\begin{aligned}
& \alpha_{3}^{*}=\alpha_{1}-\frac{Q_{12}\left(u_{m 1}+u_{m 2}\right)}{\left(s_{11}+s_{12}\right)}, \quad \alpha_{6}^{*}=-\frac{Q_{44}}{s_{44}} u_{m 6}, \\
& \alpha_{11}^{*}=\alpha_{11}+\frac{1}{2\left(s_{11}^{2}+s_{12}^{2}\right)}\left[\left(Q_{11}^{2}+Q_{12}^{2}\right) s_{11}-2 Q_{11} Q_{12} s_{12}\right],
\end{aligned}
$$

$$
\begin{aligned}
\alpha_{33}^{*}= & \alpha_{11}+\frac{Q_{12}^{2}}{s_{11}+s_{12}}, \\
\alpha_{12}^{*}= & \alpha_{12}-\frac{1}{s_{11}^{2}-s_{12}^{2}}\left[\left(Q_{11}^{2}+Q_{12}^{2}\right) s_{12}-2 Q_{11} Q_{12} s_{11}\right] \\
& +\frac{Q_{44}}{2 s_{44}}, \\
\alpha_{13}^{*}= & \alpha_{12}+\frac{Q_{12}\left(Q_{11}+Q_{12}\right)}{s_{11}+s_{12}} .
\end{aligned}
$$

Here $P_{i}(i=1,2,3)$ is the polarization component, $\alpha_{1}, \alpha_{i j}$, $\alpha_{i k j}$, and $\alpha_{i j k l}$ are the dielectric stiffness and higher-order stiffness coefficients at constant stress, $s_{m n}$ is the elastic compliance at constant polarization, and $Q_{k l}$ is the electrostrictive constant.

\section{INFLUENCE OF MISFIT STRAINS ON THE FERROELECTRIC PHASE TRANSITION}

Using the obtained potential we derived the influence of misfit strains on the temperature of ferroelectric phase transition occurring in thin epitaxial films. The types of ferro- electric phases forming just below the transition temperature were also determined. (It should be noted that during the further cooling of the film the formed ferroelectric phase can undergo further phase transformations.) Since the renormalized second-order coefficients $\alpha_{i j}^{*}$ of considered films are positive, ${ }^{9}$ the paraelectric to ferroelectric phase transition is of the second order. Such a transition is caused by the loss of the stability of a paraelectric phase and, hence, occurs if some variation in polarization components $P_{i}$ at the proximity of zero polarization may lead to decrease in the thermodynamic potential $\widetilde{G}$. It takes place if the quadratic form $\alpha_{1}^{*} P_{1}^{2}+\alpha_{2}^{*} P_{2}^{2}+\alpha_{3}^{*} P_{3}^{2}+\alpha_{6}^{*} P_{1} P_{2}$ is not positively determined and, hence, has even one negative eigenvalue. The eigenvalues $\lambda_{i}(i=1,2,3)$ of the above form can be easily obtained as

$$
\lambda_{1,2}=\frac{1}{2}\left[\left(\alpha_{1}^{*}+\alpha_{2}^{*}\right) \pm \sqrt{\left(\alpha_{1}^{*}-\alpha_{2}^{*}\right)^{2}+\alpha_{6}^{* 2}}\right],
$$

$$
\lambda_{3}=\alpha_{3}^{*} \text {. }
$$

The minimal eigenvalue defined by Eq. (9) obviously becomes negative if $4 \alpha_{1}^{*} \alpha_{2}^{*}<\alpha_{6}^{* 2}$, so the paraelectric phase loses the stability with respect to certain correlated variations in polarization components $P_{1}$ and $P_{2}$ at the temperature $T_{12}$, which can be determined from the equation $4 \alpha_{1}^{*} \alpha_{2}^{*}=\alpha_{6}^{* 2}\left(\alpha_{1}^{*}\right.$ and $\alpha_{2}^{*}$ are temperature dependent). In other words $T_{12}$ is the temperature of the second-order transformation into the ferroelectric phase with two nonzero polarization components, namely, $P_{1}$ and $P_{2}$. In its turn the temperature $T_{3}$ of the stability loss with respect to the variation in the component $P_{3}$ can be easily calculated from the equation $\alpha_{3}^{*}=0$. The ferroelectric phase forming at this temperature has only one nonzero polarization component $P_{3}$. Substituting expressions (2)-(4) for $\alpha_{i}^{*}$ into the above equations we can write down $T_{12}$ and $T_{3}$ in an explicit form as

$$
\begin{aligned}
T_{12}= & T_{0}+\varepsilon_{0} C\left[\sqrt{\frac{\left(Q_{11}-Q_{12}\right)^{2}}{\left(s_{11}-s_{12}\right)^{2}}\left(u_{m 1}-u_{m 2}\right)^{2}+\left(\frac{Q_{44} u_{m 6}}{s_{44}}\right)^{2}}\right. \\
& \left.+\frac{\left(Q_{11}+Q_{12}\right)}{\left(s_{11}+s_{12}\right)}\left(u_{m 1}+u_{m 2}\right)\right] \\
T_{3} & =T_{0}+2 \varepsilon_{0} C \frac{Q_{12}\left(u_{m 1}+u_{m 2}\right)}{\left(s_{11}+s_{12}\right)}
\end{aligned}
$$

where $T_{0}$ and $C$ are the Curie-Weiss temperature and constant, respectively, of a bulk crystal and $\varepsilon_{0}$ is the permittivity of the vacuum.

Obviously during the cooling of the heterostructure the ferroelectric phase with the highest formation temperature generates primarily. Therefore the temperature $T_{c}$ of the ferroelectric phase transformation occurring in considered films can be determined as $T_{c}=\max \left\{T_{12}, T_{3}\right\}$.

Equations (11) and (12) can be used to define the type of ferroelectric phase forming in epitaxial film just below the transition temperature $T_{c}$. Using the electrostrictive constants and elastic compliances listed in Ref. 9 we calculated the regions of formation of different ferroelectric phases for BT epitaxial films with various misfit strains. The results are shown in Figs. 1 and 2 for $u_{m 6} \neq 0$ and $u_{m 6}=0$, respectively. 


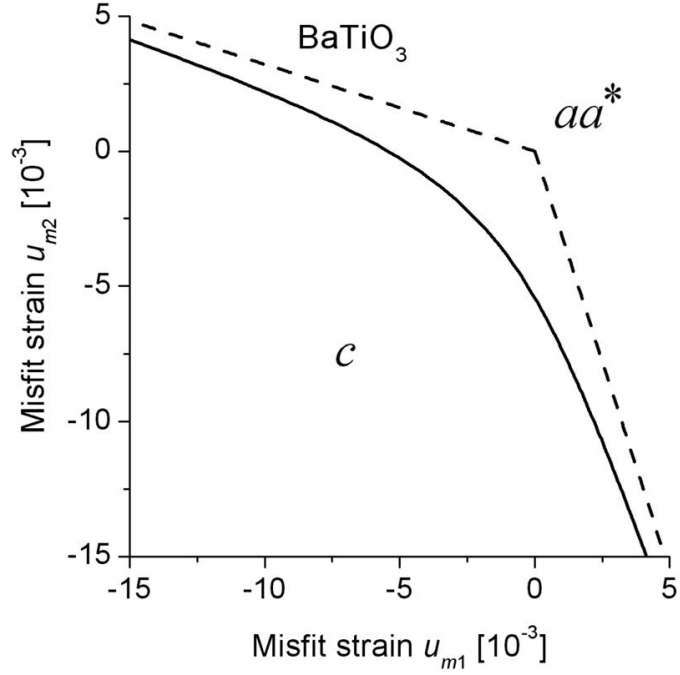

FIG. 1. Map of phases forming during the ferroelectric phase transition in BT epitaxial films under shear misfit strain $u_{m 6}=0.02$. The asymptote of a phase boundary at $u_{m 6} \rightarrow 0$ is shown by a dashed line.

In the case of nonzero shear strain $u_{m 6}$ (Fig. 1) two ferroelectric phases can form during the ferroelectric phase transformation. These are the orthorhombic $c$ phase where the vector of spontaneous polarization $\mathbf{P}_{s}$ is oriented perpendicular to the film plane and orthorhombic $a a^{*}$ phase ${ }^{19}$ where the vec-

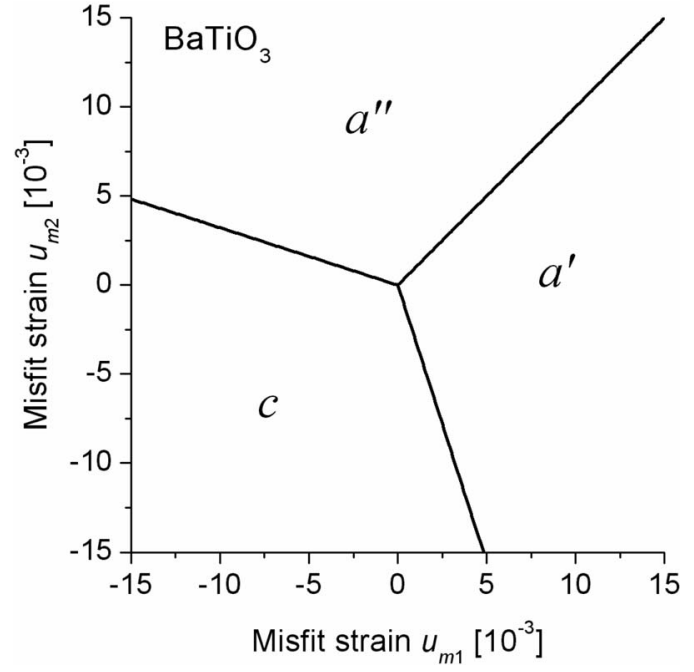

FIG. 2. Map of phases forming during the ferroelectric phase transition in BT epitaxial films under zero shear misfit strain. The phase boundaries are given by relations $u_{m 1}=u_{m 2}$ if $u_{m 1}, u_{m 2}>0 ; u_{m 2}=s_{11} / s_{12} u_{m 1}$ if $u_{m 1}>0, u_{m 2}$ $<0$; and $u_{m 2}=s_{12} / s_{11} u_{m 1}$ if $u_{m 1}<0, u_{m 2}>0$.

tor of spontaneous polarization $\mathbf{P}_{s}$ is rotated away from the in-plane face diagonal of the unit cell $\left(P_{1} \neq P_{2} \neq 0, P_{3}=0\right)$. It can be shown that the absolute values of polarization components $P_{1}$ and $P_{2}$ in the just formed $a a^{*}$ phase are linked by the relation

$$
\frac{P_{1}}{P_{2}}=\left\{\frac{\sqrt{1+\left[\frac{Q_{44}\left(s_{11}-s_{12}\right) u_{m 6}}{\left(Q_{11}-Q_{12}\right) s_{44}\left(u_{m 1}-u_{m 2}\right)}\right]^{2}}+\operatorname{sgn}\left(u_{m 1}-u_{m 2}\right)}{\sqrt{1+\left[\frac{Q_{44}\left(s_{11}-s_{12}\right) u_{m 6}}{\left(Q_{11}-Q_{12}\right) s_{44}\left(u_{m 1}-u_{m 2}\right)}\right]^{2}}-\operatorname{sgn}\left(u_{m 1}-u_{m 2}\right)}\right\}^{1 / 2} .
$$

In the case of zero shear strain $u_{m 6}$ (Fig. 2) the ferroelectric phase transformation can lead to the formation of three ferroelectric phases, namely, $c$ phase, $a^{\prime}$ phase $\left(P_{1} \neq 0, P_{2}=P_{3}\right.$ $=0)$, and $a^{\prime \prime}$ phase $\left(P_{2} \neq 0, P_{1}=P_{3}=0\right)$. The expressions for boundary lines separating the different phases in Fig. 2 can be easily obtained analytically (see caption in Fig. 2). It should be emphasized that in the considered case the character of the phase map depends only on elastic properties of the material and it is not affected by electrostrictive constants. The lines limiting the region of $c$ phase formation (Fig. 2) coincide with asymptotes of the hyperbola shown in Fig. 1. One can see that the application of shear strain irrespective of its sign always suppresses the formation of $c$ phase and favors the formation of $a a^{*}$ phase. This tendency is obviously caused by the electrostrictive coupling between the shear strain $u_{m 6}$ and in-plane polarization components $P_{1}$ and $P_{2}$.

It should be also noted that in epitaxial films under nonzero misfit strains, the ferroelectric transition can never lead to the formation of the triclinic $r^{*}$ phase with three nonzero polarization components $\left(P_{1} \neq P_{2} \neq P_{3} \neq 0\right)$. This phase may be formed only from existing ferroelectric phases during the further cooling of the film.

\section{PHASE DIAGRAMS OF $\mathrm{BaTiO}_{3}$ AND $\mathrm{Pb}(\mathrm{Zr}, \mathrm{Ti}) \mathrm{O}_{3}$ EPITAXIAL FILMS}

In this section we apply the thermodynamic potential derived in Sec. II to calculate the phase diagram of BT and PZT epitaxial films under substrate-induced anisotropic biaxial misfit strains. It should be noted that the phase diagrams have been already obtained for anisotropically strained BT epitaxial films with mechanically free ${ }^{20}$ and loaded ${ }^{25}$ surface. However, these calculations ${ }^{20,25}$ were performed on the base of six-order polynomial of Landau-Devonshire expansion, ${ }^{31}$ which can be used only for BT films under relative small compressive strains. ${ }^{30,32}$ Besides that the set of expansion coefficients used in Ref. 20 is incorrect, since on its base the sequence of phase transitions existing in free BT single crystals cannot be explained. Therefore we recalculated the phase diagram for considered BT films with the use of the thermo- 


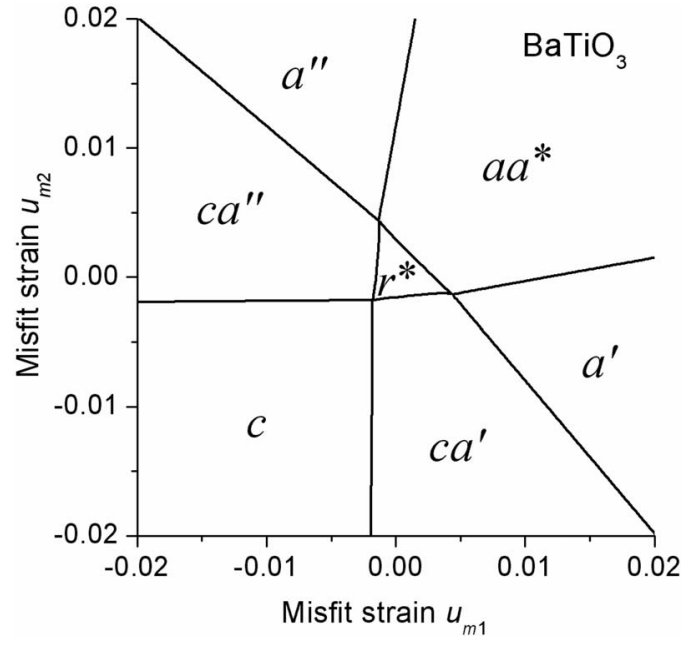

FIG. 3. Horizontal cross section of the three-dimensional (3D) phase diagram of single-domain BT films grown on orthorhombic substrates. The phase map corresponds to $T=25^{\circ} \mathrm{C}$.

dynamic potential based on an eight-order polynomial that correctly describes the ferroelectric properties of BT single crystals within the wide range of temperatures. ${ }^{30}$ In our calculations we used the dielectric stiffness coefficients given in Ref. 30 and other material parameters listed in Ref. 9.

The phase map of BT epitaxial film under zero shear strain at room temperature is shown in Fig. 3. As one can see, it differs drastically from the similar map calculated in Ref. 20. In comparison with the phase map of epitaxial film under isotropic biaxial strain, ${ }^{9,10}$ the diagram obtained for the case of nonequal misfit strains contains other ferroelectric phases. The appearance of these phases is caused by the fact that the strain anisotropy $\left(u_{m 1} \neq u_{m 2}\right)$ lowers the symmetry of the paraelectric phase from cubic to orthorhombic. Hence the symmetry of ferroelectric phases also lowers. Instead of the monoclinic $r$ phase $\left(P_{1}=P_{2} \neq 0, P_{3} \neq 0\right)$ forming in BT films grown on cubic substrates, ${ }^{10}$ the triclinic $r^{*}$ phase $\left(P_{1} \neq P_{2}\right.$ $\left.\neq P_{3} \neq 0\right)$ appears in the general case. The strain anisotropy also transforms the $a a$ phase $\left(\left|P_{1}\right|=\left|P_{2}\right| \neq 0, P_{3}=0\right)$ into the $a a^{*}$ phase $\left(P_{1} \neq P_{2} \neq 0, P_{3}=0\right)$, where $\mathbf{P}_{s}$ is rotated away from the in-plane face diagonal of the unit cell. Remarkably, when the misfit strains are opposite in sign, other ferroelectric phases appear that do not form at $u_{m 1}=u_{m 2}$. These are the $a$ phase, where the spontaneous polarization $\mathbf{P}_{s}$ is oriented along one of the in-plane edges of the prototypic unit cell and the $c a$ phase, where $\mathbf{P}_{s}$ is parallel to one of the out-ofplane faces of this cell.

We also calculated the phase diagram of $\mathrm{Pb}\left(\mathrm{Zr}_{0.50} \mathrm{Ti}_{0.50}\right) \mathrm{O}_{3}$ (PZT 50/50) epitaxial film under the same conditions as BT films (room temperature and zero shear strain $u_{m 6}$ ). In this case we kept only the terms up to the sixth order on polarization components in thermodynamic potential (1). Our calculations were carried out using the dielectric stiffness $\alpha_{1}, \alpha_{i j}$, and $\alpha_{i j k}$ at constant stress, the electrostrictive constants $Q_{k l}$ taken from Ref. 33, and the elastic compliances $s_{m n}$ at constant polarization given in Ref. 16. The obtained phase map of PZT 50/50 films is shown in Fig. 4. One can note the strong enlargement of the triclinic field in this phase diagram, which is evidently caused by the prox-

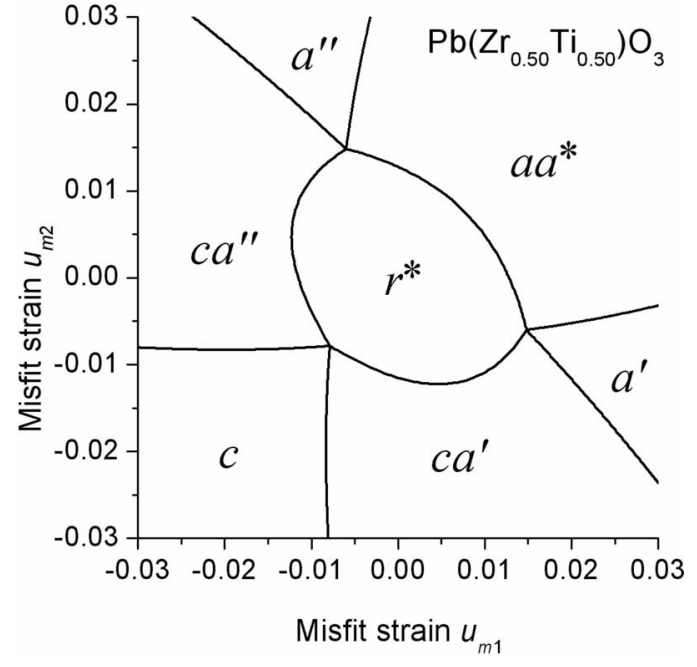

FIG. 4. Horizontal cross section of the 3D phase diagram of single-domain PZT 50/50 films grown on orthorhombic substrates. The phase map corresponds to $T=25^{\circ} \mathrm{C}$.

imity of PZT 50/50 composition to the morphotropic phase boundary. It should be also emphasized that both obtained phase maps of BT and PZT 50/50 epitaxial films differ markedly from the one of $\mathrm{PbTiO}_{3}$ (PT) films. ${ }^{19}$ In particular, at near zero misfit strains the two-dimensional clamping stabilizes the triclinic $r^{*}$ phase in BT and PZT 50/50 films, but not the tetragonal $c$ phase as in PT films.

To single out the influence of the shear strain on the phase states existing in epitaxial heterostructures at room temperature, we also calculated the phase diagrams of BT and PT thin films under isotropic biaxial strains and nonzero shear deformation. For the material parameters of PT involved in Eqs. (1)-(8) we employed the numerical values listed in Ref. 10. The phase maps of BT and PT epitaxial films are shown in Figs. 5 and 6, respectively. From these maps it is clear that the application of the shear strain favors the transitions associated with the increase in polarization components $P_{1}$ and $P_{2}$. This tendency has a natural explana-

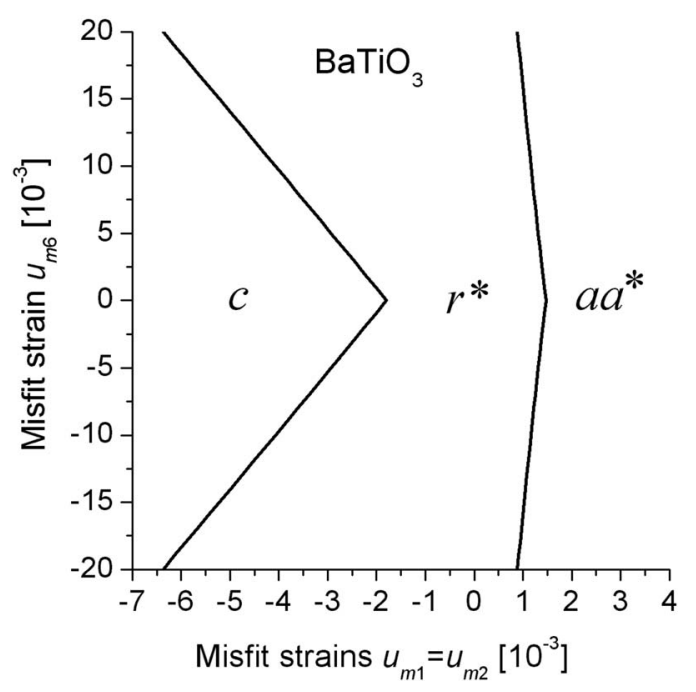

FIG. 5. Horizontal cross section of the 3D phase diagram of single-domain BT films grown on substrates inducing isotropic in-plane strains and nonzero shear strain. The phase map corresponds to $T=25^{\circ} \mathrm{C}$. 


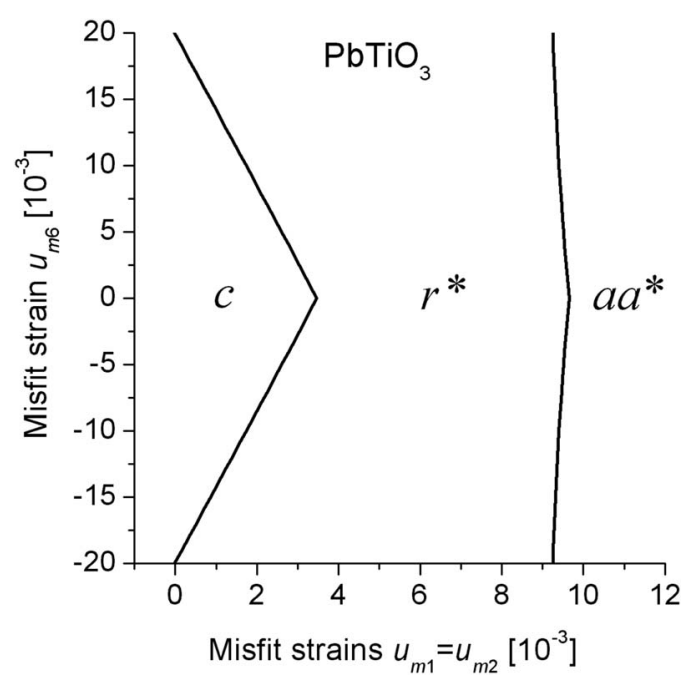

FIG. 6. Horizontal cross section of the 3D phase diagram of single-domain PT films grown on substrates inducing isotropic in-plane strains and nonzero shear strain. The phase map corresponds to $T=25^{\circ} \mathrm{C}$.

tion. Indeed, the simultaneous increase in $P_{1}$ and $P_{2}$ raises the spontaneous shear deformation of ferroelectric film in the interface plane. Since the shear stress within the film is proportional to the difference between the total shear deformation (which is dictated by the substrate) and the spontaneous shear deformation, the appearance of the latter reduces the total elastic energy of the ferroelectric layer. Therefore the increase in in-plane polarization components is a mechanism for the relaxation of internal stresses caused by the shear two-dimensional clamping.

\section{STRAIN-INDUCED ANISOTROPY OF IN-PLANE DIELECTRIC PERMITTIVITY}

In this section we apply the modified thermodynamic potential derived in Sec. I to calculate the dielectric properties of epitaxial ferroelectric films. The tensor of reciprocal dielectric susceptibility $\chi_{i j}$ of a ferroelectric phase characterized by the spontaneous polarization vector $\mathbf{P}_{\mathrm{s}}^{\mathbf{0}}$ can be expressed through the second partial derivatives of the modified thermodynamic potential as

$$
\chi_{i j}=\left.\frac{\partial^{2} \widetilde{G}}{\partial P_{i} \partial P_{j}}\right|_{\mathbf{P}=\mathbf{P}_{\mathbf{s}}^{\mathbf{0}}} .
$$

In its turn the tensor of the relative dielectric permittivity of a ferroelectric film is $\varepsilon_{i j}=\delta_{i j}+\left(\chi_{i j}\right)^{-1} / \varepsilon_{0}$, where $\delta_{i j}$ is the Kronecker delta and $\left(\chi_{i j}\right)^{-1}$ is the inverse of $\chi_{i j}$. The above expression introduces the dielectric tensor defined in the film reference frame that coincides with the crystallographic reference frame of the prototypic (paraelectric) unit cell. The dielectric permittivity $\varepsilon_{\phi}$ measured along any direction in the film plane can be expressed through the tensor transformation as

$$
\varepsilon_{\phi}=\varepsilon_{11} \cos ^{2} \phi+\varepsilon_{22} \sin ^{2} \phi+\varepsilon_{12} \sin 2 \phi,
$$

where $\phi$ is the angle between the direction of measurement and the axis $x_{1}$ of the crystallographic reference frame. Gen-

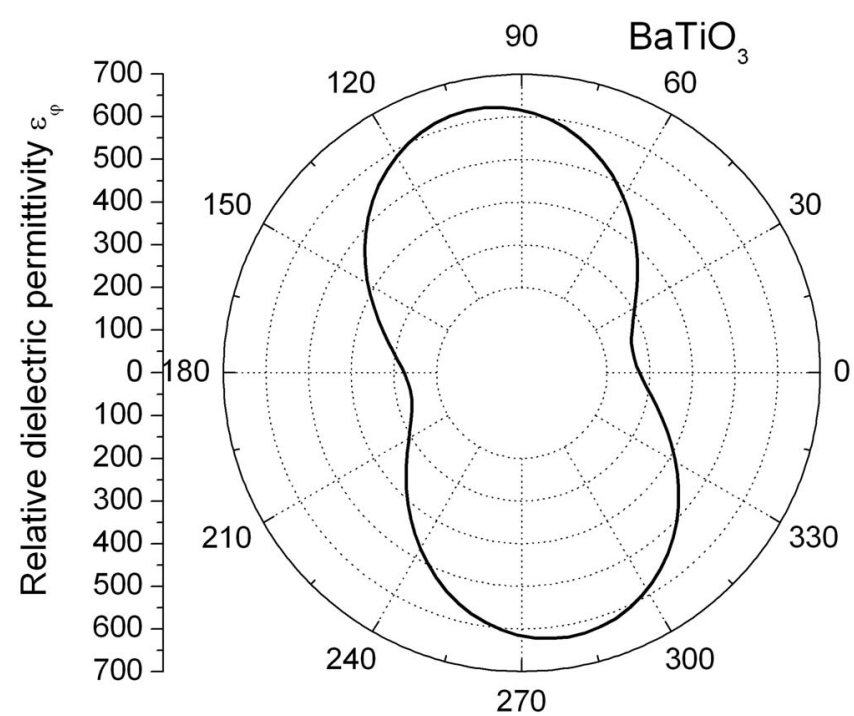

FIG. 7. Calculated directional dependence of the in-plane dielectric permittivity of epitaxial BT film. The shown polar plot corresponds to $T=25^{\circ} \mathrm{C}$, $u_{m 1}=0.003$, and $u_{m 2}=0.001$. The angle $\varphi$ is counted from the axis $x_{1}$ of a crystallographic reference frame.

erally, if $\varepsilon_{11} \neq \varepsilon_{22}$ and $\varepsilon_{12} \neq 0$ the polar plot of the function $\varepsilon_{\phi}$ is "figure of eight" with the major and minor axes rotated from the crystallographic axis $x_{1}$ by the angles $\phi_{\max }$ and $\phi_{\min }$, respectively. These angles can be easily found as a solution for equation

$$
\tan 2 \phi_{\max , \min }=\frac{2 \varepsilon_{12}}{\varepsilon_{11}-\varepsilon_{22}} .
$$

From this equation one can deduce that the major axis of "figure eight" pattern does not coincide with axis $x_{1}$ or $x_{2}$ of the crystallographic reference frame if $\varepsilon_{12} \neq 0$. This component of the dielectric permittivity tensor may be nonzero only if both in-plane polarization components $P_{1}$ and $P_{2}$ are nonzero or if the ferroelectric layer is under the shear misfit strain. At the absence of the latter the only $r^{*}$ and $a a^{*}$ phases can exhibit the above-mentioned angular dependence of the in-plane dielectric permittivity when the directions of maximal/minimal permittivity do not coincide with crystallographic axes.

In order to illustrate the obtained expressions we calculated the directional dependence of in-plane dielectric permittivity for BT epitaxial films at a room temperature and zero bias field. This dependence for the cases $u_{m 1}=0.003$, $u_{m 2}=0.001$, and $u_{m 6}=0$ is shown as a polar plot in Fig. 7. It can be seen that even small difference of misfits produces marked anisotropy of dielectric properties. Under the above misfits the BT film is in aa* crystallographic phase (see Fig. 3) so the directional dependence of in-plane permittivity is figure of eight with the major axis rotated away from any in-plane edge of the prototypic unit cell. The similar directional dependence of in-plane dielectric permittivity was observed on $\left(\mathrm{Ba}_{0.60} \mathrm{Sr}_{0.40}\right) \mathrm{TiO}_{3}$ (BST) epitaxial films with thickness $75 \mathrm{~nm}$ grown on $\mathrm{NdGaO}_{3}$ substrate that induces the anisotropic biaxial strains within the film. ${ }^{7}$

It should be noted that the ferroelectric films studied by Simon et $a .^{7}$ have $\langle 110\rangle$ crystallographic orientation and, hence, cannot be investigated with the use of potential intro- 
duced in Sec. II. Nevertheless, one can calculate the tensor of the dielectric permittivity of such films using the proper thermodynamic potential derived from Ref. 21. Applying this potential we defined the phase state of $75 \mathrm{~nm}$ BST epitaxial film ${ }^{7}$ and then obtained the directional dependence of the in-plane dielectric permittivity, which is obviously expressed by the same formulae as the one cited in the present section [Eq. (15)]. Our theoretical calculations have shown that the considered film has zero dielectric permittivity $\varepsilon_{12}$, and, hence, should not exhibit the directional dependence measured experimentally. This discrepancy may be caused by several factors. For one thing, the complete set of material parameters involved in thermodynamic potential ${ }^{21}$ is unknown for BST and even for $\mathrm{SrTiO}_{3}$. The theoretical calculations for BST (Refs. 14, 15, and 22) are usually based on the only fourth-order thermodynamic potential and approximate values of electrostrictive constants $Q_{i j}$ typical of perovskite-type oxides. ${ }^{34}$ In addition, the numerical values of misfit strains in the considered film were extracted from the plots in Ref. 8 and, hence, are approximate. Therefore our numerical calculations of the dielectric properties of BST films may be regarded only as a rough estimate.

\section{CONCLUSIONS}

In summary, the modified thermodynamic potential based on the eight-order Landau-Devonshire polynomial was derived for epitaxial ferroelectric films under the most general strain state governed by the dissimilar substrate. With the use of the obtained potential the influence of misfit strains on the temperature of ferroelectric phase transition and the properties of formed phases was studied theoretically. It was found that the application of in-plane shear strain irrespective of its sign always suppresses the formation of ferroelectric $c$ phase and raises the temperature of $a a^{*}$ phase formation. The phase diagrams of anisotropically strained BT epitaxial films were obtained in the frame of eight-order phenomenological theory, which, in contrast with the sixth-order one, is physically correct in the whole range of considered misfit strains. The significant feature induced by in-plane shear strains is the shift of the points of misfitstrain-driven phase transitions toward the phases with smaller out-of-plane polarization. On the base of obtained thermodynamic potential the directional dependence of inplane dielectric permittivity of an epitaxial film was derived. It was established that the anisotropy of in-plane strains causes the figure of eight shape of this dependence. The rotation of its major axis from the crystallographic axes of the film is due to the certain anisotropy of in-plane strains or due to the application of the shear strain. Such a behavior of the directional dependence coincides qualitatively with the one obtained in experiments.

\section{ACKNOWLEDGMENTS}

The research described in this publication was made possible in part by the Deutsche Forschungsgemeinschaft, Germany (Grant No. 436 RUS 17/71/06).

${ }^{1}$ C. M. Foster, G.-R. Bai, R. Csencsits, J. Vetrone, R. Jammy, L. A. Wills, E. Carr, and J. Amano, J. Appl. Phys. 81, 2349 (1997).

${ }^{2}$ T. Tybell, C. H. Ahn, and J.-M. Triscone, Appl. Phys. Lett. 75, 856 (1999).

${ }^{3}$ N. Yanase, K. Abe, N. Fukushima, and T. Kawakubo, Jpn. J. Appl. Phys., Part 1 38, 5305 (1999).

${ }^{4}$ S. K. Streiffer, J. A. Eastman, D. D. Fong, C. Thompson, A. Munkholm, M. V. Ramana Murty, O. Auciello, G. R. Bai, and G. B. Stephenson, Phys. Rev. Lett. 89, 067601 (2002).

${ }^{5}$ M. Okano, Y. Watanabe, and S.-W. Cheong, Appl. Phys. Lett. 82, 1923 (2003).

${ }^{6}$ V. Nagarajan, S. Prasertchoung, T. Zhao, H. Zheng, J. Ouyang, R. Ramesh, W. Tian, X. Q. Pan, D. M. Kim, C. B. Eom, H. Kohlstedt, and R. Waser, Appl. Phys. Lett. 84, 5225 (2004).

${ }^{7}$ W. K. Simon, E. K. Akdogan, A. Safari, and J. A. Bellotti, Appl. Phys. Lett. 87, 082906 (2005).

${ }^{8}$ W. K. Simon, E. K. Akdogan, and A. Safari, J. Appl. Phys. 97, 103530 (2005).

${ }^{9}$ N. A. Pertsev, A. G. Zembilgotov, and A. K. Tagantsev, Phys. Rev. Lett. 80, 1988 (1998).

${ }^{10}$ N. A. Pertsev, A. G. Zembilgotov, and A. K. Tagantsev, Ferroelectrics 223, 79 (1999).

${ }^{11}$ Y. Watanabe, Phys. Rev. B 57, 789 (1998).

${ }^{12}$ A. L. Roytburd, S. P. Alpay, V. Nagarajan, C. S. Ganpule, S. Aggarwal, E. D. Williams, and R. Ramesh, Phys. Rev. Lett. 85, 190 (2000).

${ }^{13}$ Y. L. Li, S. Y. Hu, Z. K. Liu, and L. Q. Chen, Appl. Phys. Lett. 78, 3878 (2001).

${ }^{14}$ Z.-G. Ban and S. P. Alpay, J. Appl. Phys. 91, 9288 (2002).

${ }^{15}$ Z.-G. Ban and S. P. Alpay, J. Appl. Phys. 93, 504 (2003).

${ }^{16}$ N. A. Pertsev, V. G. Kukhar, H. Kohlstedt, and R. Waser, Phys. Rev. B 67, 054107 (2003)

${ }^{17}$ J. Junquera and Ph. Ghosez, Nature (London) 422, 506 (2003).

${ }^{18}$ O. Diéguez, S. Tinte, A. Antons, C. Bungaro, J. B. Neaton, K. M. Rabe, and D. Vanderbilt, Phys. Rev. B 69, 212101 (2004).

${ }^{19}$ A. G. Zembilgotov, N. A. Pertsev, U. Böttger, and R. Waser, Appl. Phys. Lett. 86, 052903 (2005).

${ }^{20}$ J. Wang and T.-Y. Zhang, Appl. Phys. Lett. 86, 192905 (2005).

${ }^{21}$ A. G. Zembilgotov, U. Böttger, and R. Waser, Appl. Phys. Lett. 90, 036101 (2007).

${ }^{22}$ G. Akcay, I. B. Misirlioglu, and S. P. Alpay, J. Appl. Phys. 101, 104110 (2007).

${ }^{23}$ H.-X. Cao, V. C. Lo, and Z.-Y. Li, J. Appl. Phys. 101, 014113 (2007).

${ }^{24}$ Y. M. Tao and Y. Z. Wu, J. Appl. Phys. 101, 024111 (2007).

${ }^{25}$ J. H. Qiu and Q. Jiang, J. Appl. Phys. 101, 034110 (2007).

${ }^{26}$ Y. Lin, X. Chen, S. W. Liu, C. L. Chen, J.-S. Lee, Y. Li, Q. X. Jia, and A. Bhalla, Appl. Phys. Lett. 84, 577 (2004).

${ }^{27}$ Y. Lin, X. Chen, S. W. Liu, C. L. Chen, J.-S. Lee, Y. Li, Q. X. Jia, and A. Bhalla, Appl. Phys. Lett. 86, 142902 (2005).

${ }^{28}$ B. S. Kwak, A. Erbil, J. D. Budai, M. F. Chisholm, L. A. Boatner, and B. J. Wilkens, Phys. Rev. B 49, 14865 (1994).

${ }^{29}$ M. J. Haun, E. Furman, S. J. Jang, H. A. McKinstry, and L. E. Cross, J. Appl. Phys. 62, 3331 (1987).

${ }^{30}$ Y. L. Li, L. E. Cross, and L. Q. Chen, J. Appl. Phys. 98, 064101 (2005).

${ }^{31}$ A. J. Bell and L. E. Cross, Ferroelectrics 59, 197 (1984).

${ }^{32}$ It can be easily shown that ferroelectric $c$ phase formed in the case of isotropic compressive strains has a spontaneous polarization given by $P_{3}^{2}$ $=\left(-\alpha_{33}^{*} \pm \sqrt{\alpha_{33}^{* 2}-3 \alpha_{3}^{*} \alpha_{111}}\right) / 3 \alpha_{111}$. Such a solution obviously exists only if $\alpha_{33}^{* 2}>3 \alpha_{3}^{*} \alpha_{111}$. Using this inequality and dependencies of expansion coefficients on temperature (Ref. 31) we calculated the threshold value of compressive strains that equals approximately to $-0.5 \%$. At lower strains the solution for $P_{3}$ can be found not in the whole range of existence of $c$ phase.

${ }^{33}$ M. J. Haun, E. Furman, S. J. Jang, and L. E. Cross, Ferroelectrics 99, 13 (1989), Secs. I-V.

${ }^{34}$ T. Yamada, J. Appl. Phys. 43, 328 (1972). 